\title{
Heartbeat: Intervention for asymptomatic severe aortic stenosis?
}

There is no question that aortic valve replacement (AVR) improves survival and reduces symptoms in symptomatic adults with severe aortic stenosis (AS); this recommendation in both European Society of Cardiology and American Heart Association/American College of Cardiology guidelines is supported by high-quality clinical trials, observational data and clinical experience. However, it remains controversial whether AVR would improve long-term outcomes in adults with severe AS who have not yet developed symptoms. Given the absence of a randomised clinical trial to address this question, Lim et al (see page 268) performed a meta-analysis of four observational studies (with a total of 1300 patients) that compared AVR at symptom onset to AVR in the absence of symptoms in adults with severe AS. The pooled data showed no significant difference between an early AVR versus symptom-driven AVR approach in cardiac mortality (OR $0.78, \mathrm{p}=0.85$ ) or sudden death (OR 0.34, $\mathrm{p}=0.25$ ). There was a trend toward reduced overall mortality with early AVR using a random effects model, which is the most appropriate statistical approach given heterogeneity between studies (figure 1). The authors conclude, "We do not believe the data are sufficient to change clinical practice until more robust, randomised data are available."

In the accompanying editorial, Marechaux and Tribouilloy (see page 258) remind us that patients may not recognise the onset of symptoms because of lifestyle adaptation to a slowly progressive disease. Thus, exercise testing "should be performed whenever possible to detect patients who are not 'truly' asymptomatic and who should undergo AVR in the presence of a reasonable operative risk". In addition, guidelines currently do recommend AVR in some patients with asymptomatic severe AS; specifically, those with a left ventricular ejection fraction $<50 \%$, an exercise test that elicits

Division of Cardiology, University of Washington, Seattle, USA

Correspondence to Professor Catherine M Otto, Division of Cardiology, Box 356422, University of Washington, Seattle, WA, USA; cmotto@u.washington. edu

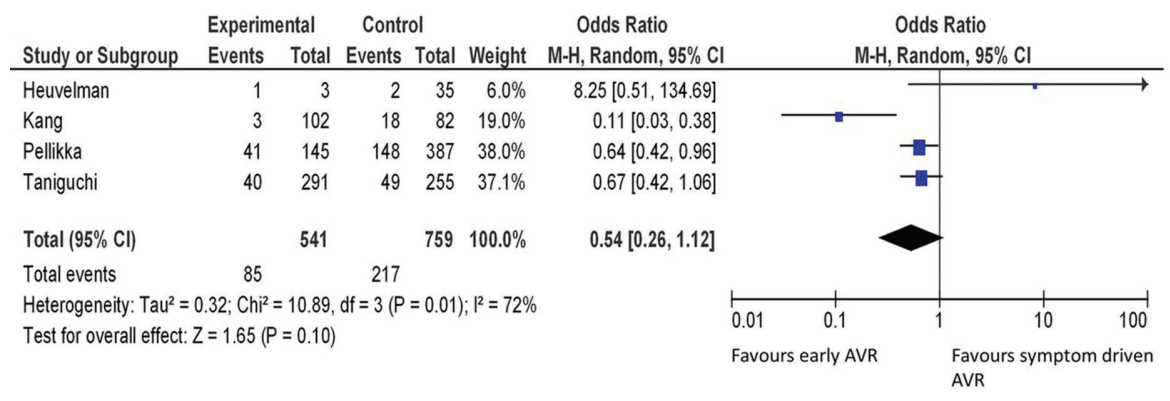

Figure 1 Random-effects model comparing all-cause mortality between patients with asymptomatic severe aortic stenosis undergoing early aortic valve replacement (AVR) and patients on a symptomdriven AVR protocol.

symptoms or a fall in blood pressure, very severe AS (defined variability as an aortic velocity over 5.0 or $5.5 \mathrm{~m} / \mathrm{s}$ ), or rapidly progressive obstruction with severe valve calcification. In other patients, the potential benefits of early AVR must be balanced against the risks of intervention and prosthetic valve complications. Randomised trials are in progress; in the meanwhile, "a tailor-made management by a heart team in specialised heart valve clinics is recommended in patients with asymptomatic severe AS, with thorough risk stratification, patient education and close follow-up."

There is little data on long term outcome of patients with congenital heart disease who underwent corrective surgery in infancy and childhood. In this issue of Heart, Cuypers et al (see page 273) report outcomes in 53 patients with congenital valvular pulmonic stenosis followed for an average of 37 years after corrective surgery. At 40 years, overall survival was
$94 \%$ with an event free survival of $68 \%$ (figure 2). Repeat intervention was needed in $25 \%$, mainly for residual stenosis, typically within 3 years and for pulmonic regurgitation, typically 20 years after the initial procedure. Although only $13 \%$ had right ventricular dysfunction, left ventricular systolic dysfunction was present in $41 \%$ of patients.

In an editorial, d'Udekem (see page 260) points out that surgical treatment for congenital pulmonic valve stenosis was replaced by transcatheter balloon dilation over 30 years ago and that outcomes with balloon dilation may differ from those with surgical intervention. However, this study does provide important data about long term outcomes after congenital heart surgery; in particular, the observation that late LV dysfunction was common is of particular concern. The mechanism for LV dysfunction is not clear: "Whether these differences are due to the trauma related to surgery or the developmental

\section{Survival of data 1:Survival proportions}

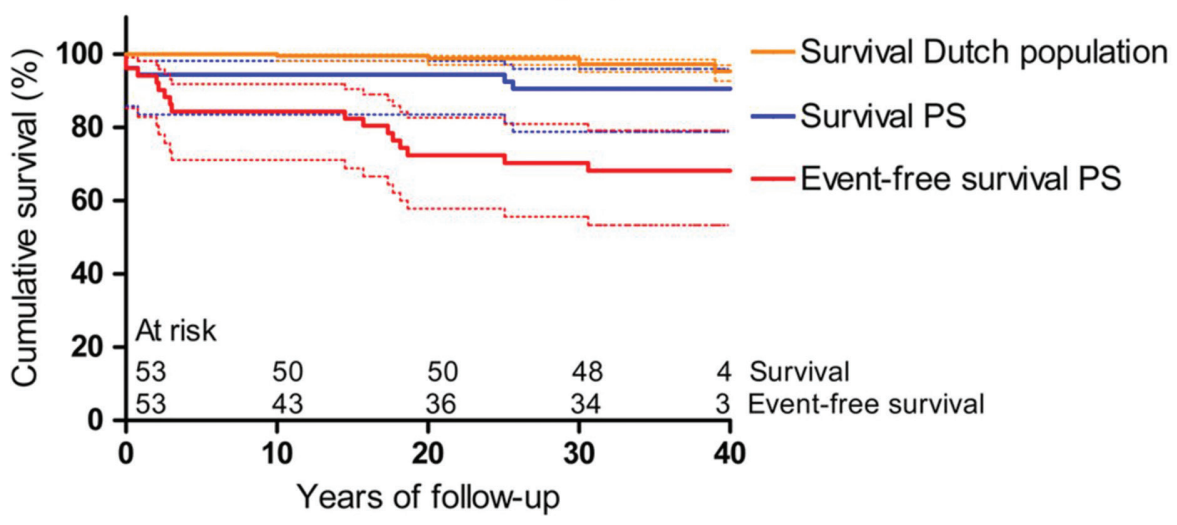

Figure 2 Survival and event-free survival of patients with pulmonary stenosis (PS) compared with the general Dutch population. 


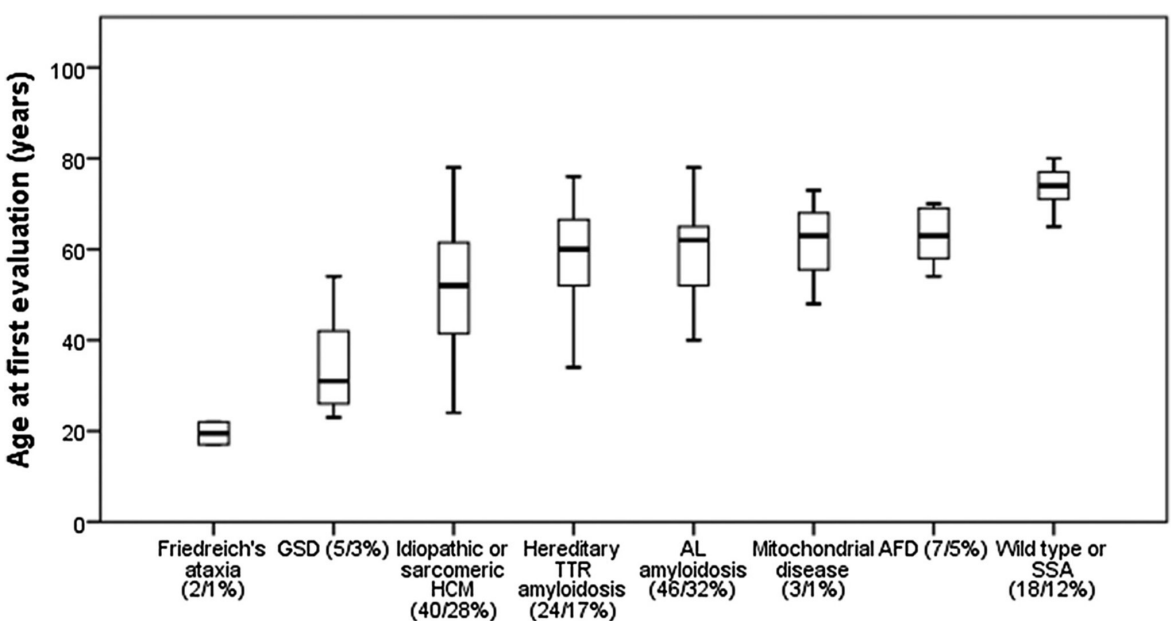

Figure 3 Age at first evaluation according to aetiology in patients with left ventricular (LV) systolic dysfunction. Distribution of median age at first evaluation according to aetiology in patients with LV systolic dysfunction (145 patients The box represents the IQR, and the line across the box indicates the median. The whiskers represent the highest and lowest values, which are no greater than 1.5* IQR from the upper or lower edge of the box. In parentheses, number of patients in each group/percentage. AFD, Anderson-Fabry disease; AL, immunoglobulin light chain; GSD, glycogen storage disease; HCM, hypertrophic cardiomyopathy; SSA, senile systemic amyloidosis; TTR, transthyretin type.

factors remains to be elucidated." With respect to the effects of chronic pulmonic valve regurgitation, he suggests "This manuscript seems to point to the relative innocuity of isolated pulmonary regurgitation and one can wonder whether we

should not veer away from the techniques of valve preservation until their efficiency is better demonstrated."

Although the majority of patients diagnosed with hypertrophic cardiomyopathy (HCM) will have normal or

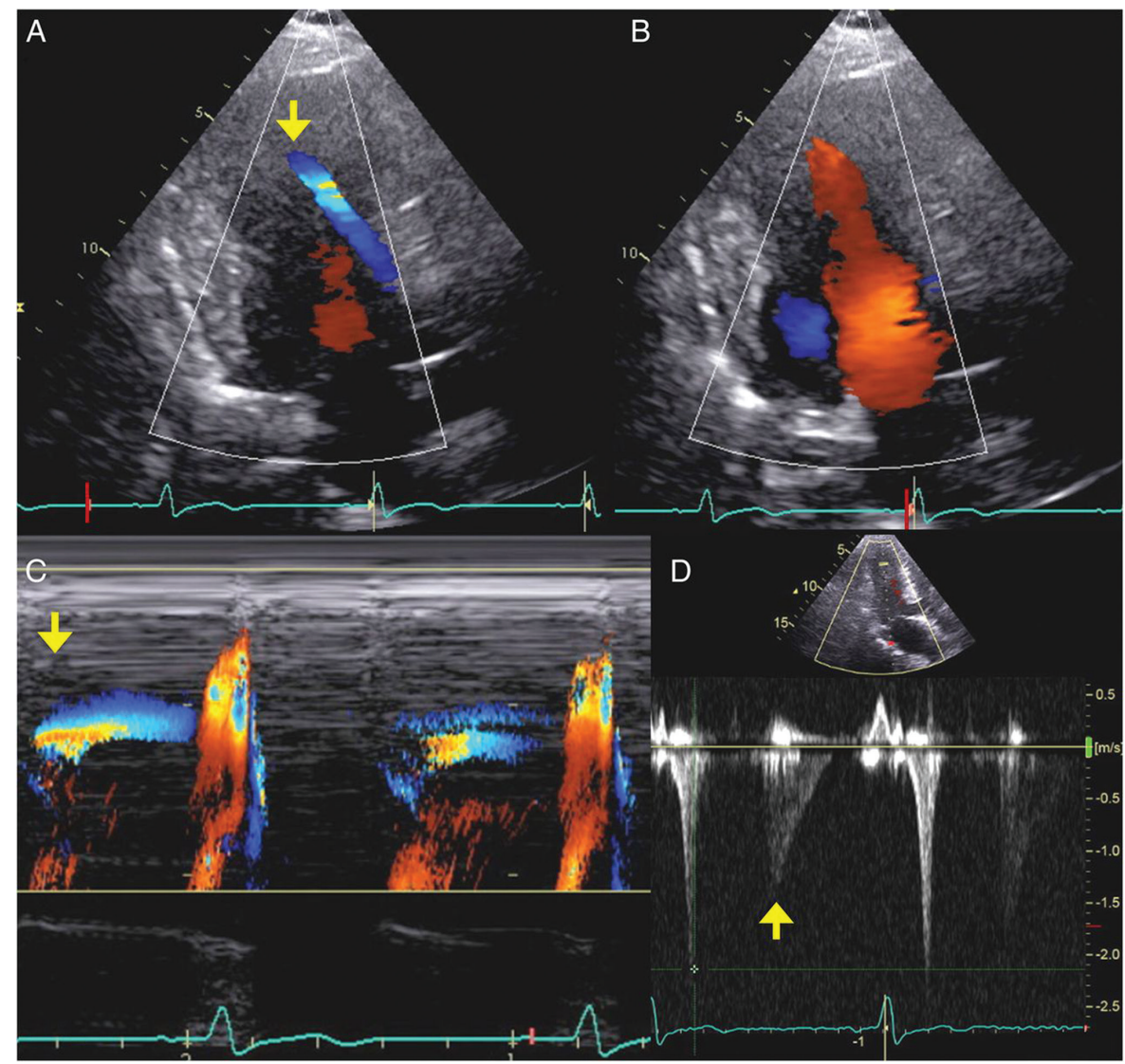

Figure 4 Transthoracic echocardiogram. Apical long-axis two-dimensional colour Doppler of the left ventricle in early (A) and late diastole (B). Colour M-mode of left ventricle (C). Pulsed-wave Doppler of mid left ventricle (D). hypercontractile left ventricular (LV) systolic function, as clinicians we occasionally encounter HCM patients who present with or develop LV systolic dysfunction. The underlying reasons for such differences in presentation are poorly understood. In this issue, Rosmini et al (see page 300) analyse data from two European cohorts involving 1697 patients with detailed genotypic and phenotypic assessment. They show that 'the prevalence of severe LV systolic impairment was nine times higher in patients with rare phenocopies compared to patients with idiopathic or sarcomeric HCM'. They further show that those specific types of rare phenocopies that present with LV systolic dysfunction are more common in older age. By contrast, LV dysfunction in idiopathic or sarcomeric HCM was rare with no specific age range at which it was more common (figure 3). Putting these findings together, they conclude that "systolic LV dysfunction in patients with HCM is a diagnostic clue that should prompt a systematic search for rarer phenocopies informed by the age of the patient at first presentation."

The Education in Heart article in this issue (see page 316) addresses issues related to coronary revascularisation in octogenarians. Elderly patents are an important high risk subgroup due to a high burden of comorbidities in combination with atypical symptoms, often delaying diagnosis and initiation of treatment. Although elderly patients are at higher risk of complications with coronary revascularisation (either percutaneous or surgical) they also tend to be undertreated in clinical practice and under-represented in clinical trials.

In the Image challenge (see page 324) color Doppler images (figure 4) are shown in a 77-year-old woman who presented with syncope. See if you can make the diagnosis; then check the online answer and additional imaging to check if you are right!

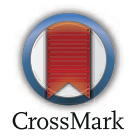

To cite Otto CM. Heartbeat: Intervention for asymptomatic severe aortic stenosis? Heart 2017; 103:253-254.

Heart 2017;103:253-254.

doi:10.1136/heartjnl-2017-311204 